\title{
Effectiveness of Smartphone Application in Histology Practice
}

\author{
Kiwook Yang $\mathbb{D}^{1}$, Sang-Uk Park $\mathbb{D}^{2}$, Jeong-Yeob Kim $\mathbb{D}^{2}$, Hyunsu Lee $\mathbb{D}^{1}$, \\ In-Jang Choi $\mathbb{D}^{1}$, Jae-Ho Lee $\mathbb{D}^{1}$ \\ ${ }^{1}$ Department of Anatomy, School of Medicine, Keimyung University \\ ${ }^{2}$ Medical Course, School of Medicine, Keimyung University
}

\begin{abstract}
In recent years, the use of smartphone applications for education is increasing. Although applications are being used for various educational aspects such as exams, the study of medical educational application is lacking. This study aims to investigate the effect of educational applications on medical education by analyzing the utility of and satisfaction with educational applications for histology courses in medical schools. The researcher employed an application called "BAND" that can be used to upload and share microscopic figures taken during histology lectures. A total of 82 first-grade students from Keimyung University Medical School who participated in this study expressed satisfaction with using "BAND". A total of 68 (83\%) students were satisfied with learning histology through the application, and $72(88 \%)$ students indicated that using the application was greatly helpful for the organizational practice test and expressed overall satisfaction with the application use. This benefit was because of easy contact (29.9\%), searching ability (29.9\%), and memory with humorous methods by friends (20.7\%). And it application is most helpful during epithelium section (20.8\%) and student want to use this application for anatomy (40.3\%) or microbiology (38.8\%) practices. The study results imply the need for further research so that the educational application can be used in various medical education fields in the future.
\end{abstract}

Keywords : Smartphone, Application, Histology, Medical application

\section{INTRODUCTION}

As the digital generations have grown up with hightech gadgets and become avid users of mobile phones and applications, smartphones have become the most important element in modern life [1]. With the rapid spread of smartphones and their growing prevalence in everyday life,

The author(s) agree to abide by the good publication practice guideline for medical journals.

The author(s) declare that there are no conflicts of interest.

Received: February 24, 2019; Revised: March 13, 2019; Accepted: March 15, 2019

Correspondence to: Jae-Ho Lee (Department of Anatomy, School of Medicine, Keimyung University, 2800, Dalgubeoldaero, Dalseo-Gu, Daegu, Republic of Korea)

E-mail: anato82@dsmc.or.kr many applications are being developed. In the past, applications have been deviated from those limited to photography, games, messaging, and social networking. Recently, applications are being created in various fields such as health and exercise are being created. As part of this trend, educational applications are also being actively developed $[2,3]$. Although these educational applications were originally aimed at infants and children in the early stages of education, they have gradually expanded to be used in higher education or linked to smart education $[4,5]$.

However, despite the development of a variety of educational applications, the study of medical educational application is lacking. Because of its nature, students of medicine acquire a vast amount of highly specialized 
knowledge, which they must then apply to the patient's treatment and diagnosis. Therefore, the reliability and validity of the content always need be verified. For example, histology requires students to observe various tissue specimens through a microscope and remember their structure and shape. In the case of microbiology, the shape of the colonies of various bacteria, the structure observed through the microscope, and the results of the tests to identify the bacteria are photographed.

Medical education should be prepared with extensive and specialized knowledge and various pictures. Therefore, educational applications can assist in learning using images and previous studies about "Facebook" and "Twitter" have been reported [6,7]. However, these applications do not guarantee individual privacy. However, in the "BAND", the members must be approved by the professors to be active member, and it can guarantee the secret of the shared content a little more.

In present study, the effect of smartphone application on medical education, especially histology, was investigated in medical students. Of many applications, "BAND" was used because of its widespread use in Korea, privacy protection, and searching function. Its satisfaction, engagement and activities were analyzed and discussed the direction for educational application for medicine.

\section{METHODS}

In order to investigate the satisfaction of medical students with the use of "BAND" in learning, a survey was conducted for a limited sample of 82 first-grade medical students (Fig. 1). We analyzed the students' satisfaction, engagement, and activities using "BAND".

The satisfaction with the application was evaluated through surveys with a valuation of the application itself and how its use improved their grades on the organizational practice test. The five-point Likert-scale survey included satisfaction statements with 1 "not helpful," 2 "slightly helpful," 3 "moderately helpful," 4 "mostly helpful," and 5 "very helpful" as options.

This study was conducted immediately after the histological learning period (March 2016 April 2016), and results were divided into the satisfaction and usefulness level, activity frequency, effectiveness, and engagement degree of the "BAND" application.

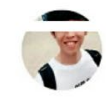

Eosinophil resembling Deadpool! Deadpool has two eyes and two eosinophil nuclei !!

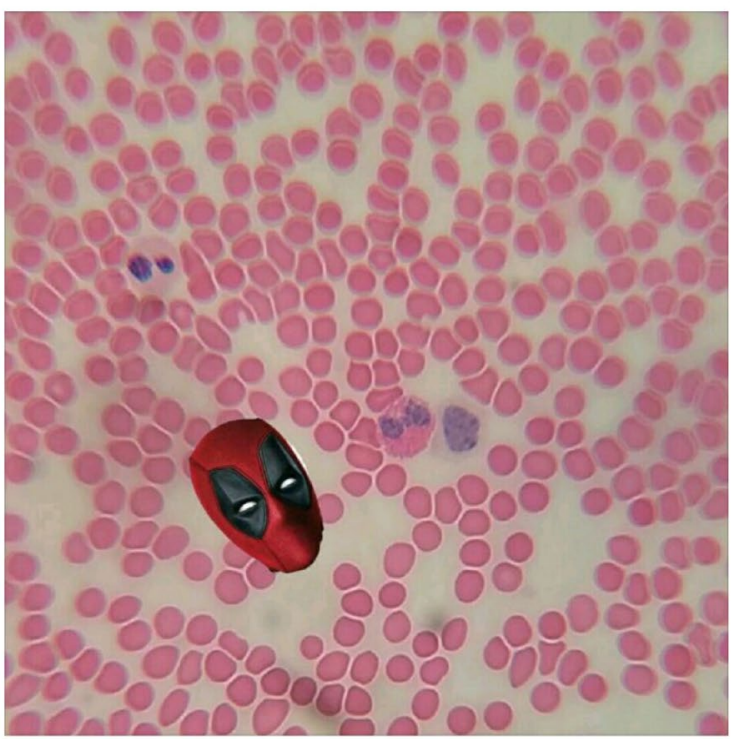

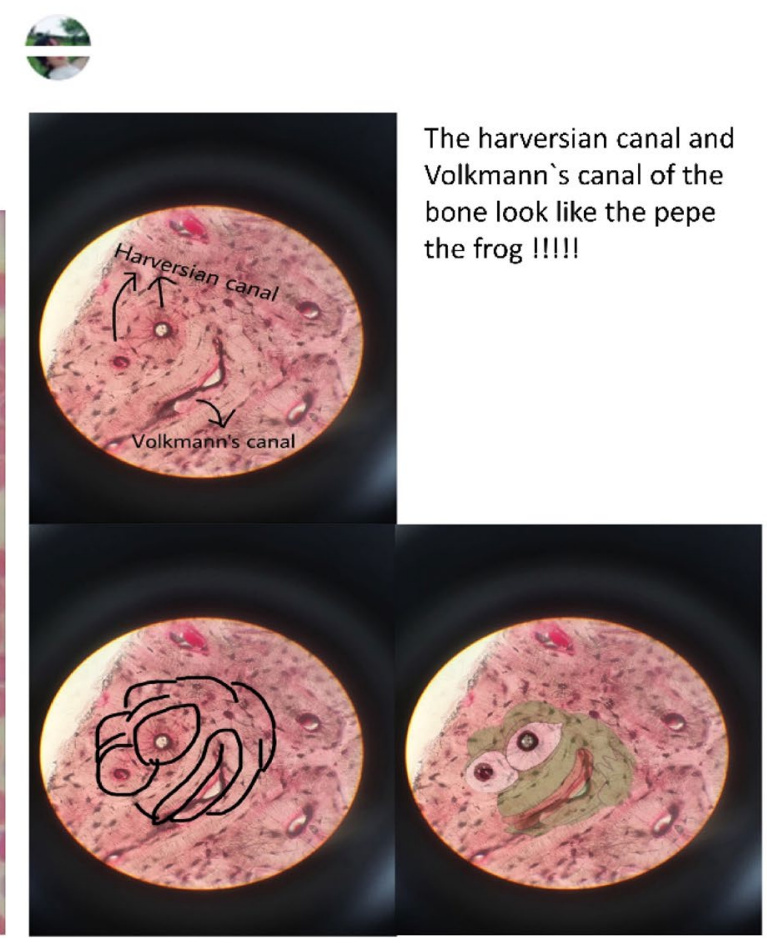

Fig. 1. Representative example of posting in educational application for Histology. 
All statistical analyses were performed using IBM SPSS statistics for Windows, V.20 (IBM Corp). Mann-Whitney U test and Kruskal-Wallis test were used to analyze the association between variables. A $P$-value of $<0.05$ was considered statistically significant.

\section{RESULTS}

The overall satisfaction, usefulness, engagement, and activity of using "BAND" for medical education were analyzed for 82 first-grade students in medical school. All the participants used the educational application "BAND" and were able to exchange information with others in the grade.

The satisfaction and usefulness of histology application were presented in Table 1. A total of 41 students (50.0\%) responded that they found the application to be very satisfied, followed by an overall majority of 27 students (33\%) who expressed mostly satisfied with its use for histology learning. When satisfaction was scored on a five-point scale, the average satisfaction score was 4.29. The usefulness for improving the scores of the organizational practice test was then analyzed. A total of 44 students $(53.7 \%)$ answered that it was very useful, and 28 students $(34.1 \%)$ said that it was mostly useful. Only 5 students $(6.1 \%)$ answered that it was slightly useful or not at all useful. It did not different ac-

Table 1. The satisfaction and usefulness of educational application for Histology.

\begin{tabular}{lcc}
\hline & Satisfaction $(\mathrm{N}, \%)$ & Usefulness $(\mathrm{N}, \%)$ \\
\hline Total & $82(100)$ & $82(100)$ \\
Very & $41(50.0)$ & $44(53.7)$ \\
Mostly & $27(32.9)$ & $28(34.1)$ \\
Moderately & $12(14.6)$ & $5(6.1)$ \\
Slightly & $1(1.2)$ & $3(3.6)$ \\
Not & $1(1.2)$ & $2(2.4)$ \\
\hline
\end{tabular}

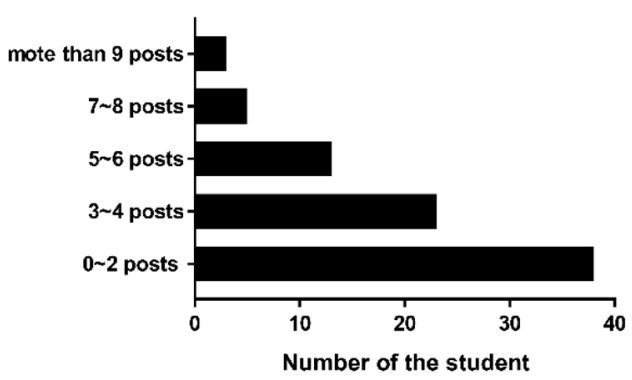

cording to gender and age.

The engagement with the "BAND" and level of activity was analyzed by counting posts and comments. The number of posts on the grade for "BAND" was analyzed and presented in Fig. 2. Most students $(38,46 \%)$ answered that they had written $0 \sim 2$ posts during the study period. Only 3 students (4\%) wrote more than 9 posts. After the students' posting frequency, the number of comments was analyzed. And, 29 students (35\%) answered that they wrote more than 9 comments, and $22(27 \%)$ answered that they wrote $5 \sim 6$ comments. A total of $8(10 \%)$ students answered that they wrote $0 \sim 2$ comments.

The students were asked about which of the 15 histological lab units they thought was made most easy to learn by the "BAND" (Fig. 3). A total of 32 (20.8\%) students answered that the "BAND" was most helpful for learning about the Epithelial tissue unit, and similar result was found in other lectures. The connective tissue unit was least helpful $(4 / 154,2.6 \%)$. And the usefulness of the application for subjects other than the histology course was investigated (Fig. 4).

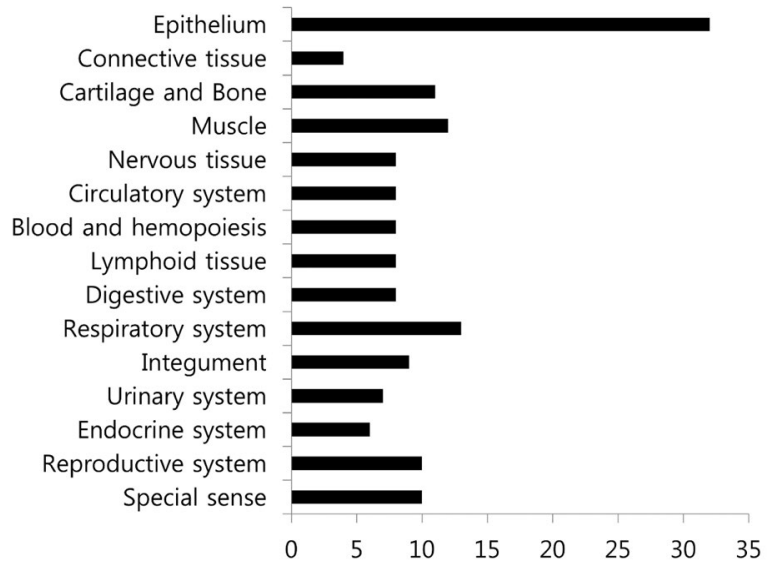

Fig. 2. The frequency of posts and comments in educational application for Histology.

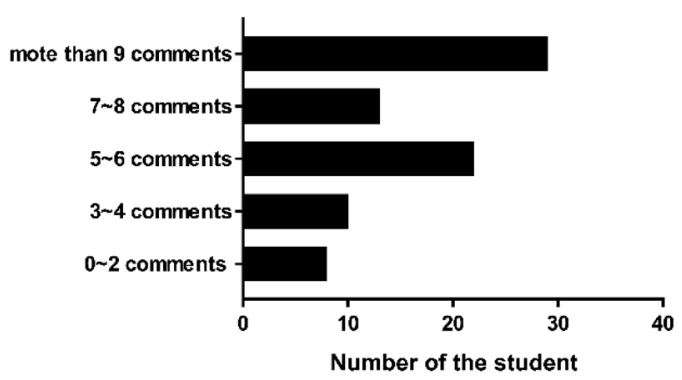

Fig. 3. The useful unit in Histology by educational application. 


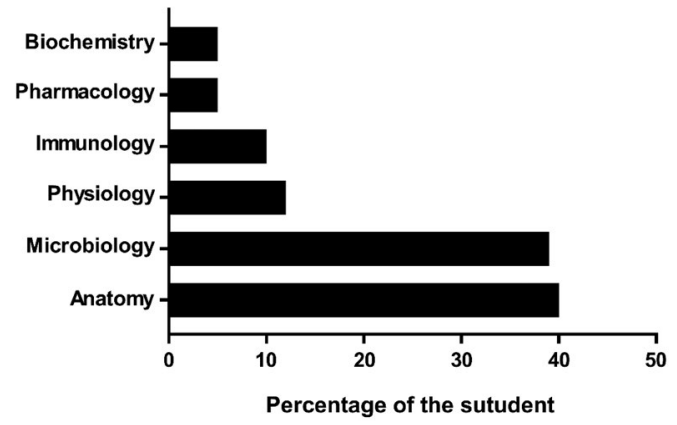

Fig. 4. The useful subject by educational application.

The analysis revealed that using the educational application for anatomy practice (40\%) and microbiology practice (39\%) was preferable. The other preferred subjects were physiology practice $(12 \%)$, immunology practice $(10 \%)$, pharmacology practice $(5 \%)$, and biochemistry practice $(5 \%)$.

\section{DISCUSSION}

In previous study on satisfaction of medical students with medical education, the average score of the questionnaire with a scale of $0 \sim 4$ points was 2.18 points, and the degree of satisfaction was moderate [8]. The present results indicate that $41(50 \%)$ and $27(33 \%)$ students found the application to be very helpful and helpful for histology learning respectively. The average satisfaction score was 4.29 , which is much higher than the satisfaction level of the previous studies on general college students and medical students [8]. It is certainly not accurate compare these results with the results of a survey with uncontrolled variables such as the nature of the course being taken or the lecturer or learner. However, even if we compare only those figures, it can be seen that the use of educational applications significantly contributes to the improvement of educational satisfaction.

Previous studies that have employed educational applications in various fields of study have demonstrated significant improvement in students' satisfaction and learning outcomes [9]. In a study of college students, $80 \%$ of the participants reported that educational applications were effective in learning. This is similar to the fact that $88 \%$ of the present participants indicated that the application was helpful in preparing for histology study and the practical exam.

The survey results indicated that a large number of students primarily used lecture textbooks or lecture books or internet searches to view lectures and practical images. However, they indicated that books or lectures were not useful when searching for desired materials. And internet browsing was convenient, but the images obtained had poor reliability. Therefore, smartphone application was both convenient and reliable showing high satisfaction score. It is therefore imperative to use educational applications in various medical subjects and practice classes that need to learn via and interact with various images, as this can significantly improve learning efficiency, satisfaction, and learning outcomes.

In addition, many participants indicated that they were able to memorize easily owing to the clever phrasings used by other students. In fact, this type of learning method has been reported by previous studies [10-12]. Peer learning refers to students learning from each other without a dominant authority figure and recognized to be more effective than traditional teaching methods improving the academic achievement of students [10-12]. Students can engage in peer learning by posting through educational applications and asking questions and sharing opinions with each other. Peer learning becomes more effective if posts are interesting or well-received by students. This shows that educational applications can make learners collaborate and simultaneously improve their academic achievement.

However, most students did not perform posting or writing activity because of passive attitude by traditional Korean education and manner. Only comment and reply activities were relatively more common. Therefore, we analyzed the reasons for this by asking additional questions to those students who wrote fewer posts. Students did not post because of the burden of interest and fun. Although writing funny posts is interesting, we should encourage students to post good photographs and sensible postings in order to increase engagement rate. To promote the participation, we give active and improved students small gift (stationery) using statistical function of BAND. Another limitation in using the application was frequent alarm about new posts and birthday of members. It is because BAND is not intended for educational used. Therefore, this alarm function was deleted.

Student selected most helpful chapter as Epithelium, because it is first histologic practice. Microscopic equipment and image were unfamiliar at that time, this application will be a great help to Histology practice. Similar responses were found in other chapter except Connective tissue. And 
student selected most helpful subject as Anatomy and Microbiology practices. It is originated from the importance of the image in these subjects. However, SNS of Anatomy image may make ethical problem, its ability should be reconsidered. Therefore, Anatomical application with closed community software should be needed.

\section{CONCLUSION}

In this study, we evaluated educational characteristics of smartphone application for Histology. Medical education is extremely specialized and important, educational development and change should be performed carefully. Therefore, it is necessary to invent an application that fulfills the needs of medical education in the future while being a satisfactory experience for the students. This should help improve the learning efficiency and academic achievements of medical students.

\section{CONFLICT OF INTEREST}

The authors have no conflict of interest to declare.

\section{REFERENCES}

1. Andrews S, Ellis DA, Shaw H, Piwek L. Beyond self-report: tools to compare estimated and real-world smartphone use. PLoS One. 2015; 10:e0139004.

2. Payne KB, Wharrad H, Watts K. Smartphone and medical related App use among medical students and junior doctors in the United Kingdom (UK): a regional survey. BMC Med Inform Decis Mak. 2012; 12:121.

3. Martin F, Ertzberger J. Here and now mobile learning: An experimental study on the use of mobile technology. Computers \& Education. 2013; 68:76-85.

4. Papadakis S, Kalogiannakis M, Zaranis N. Educational apps from the Android Google Play for Greek preschoolers: A systematic review. Computers \& Education. 2018; 116:139-60.

5. Davies BS, Rafique J, Vincent TR, Fairclough J, Packer MH, Vincent R, et al. Mobile Medical Education (MoMEd)-how mobile information resources contribute to learning for undergraduate clinical students-a mixed methods study. BMC Medical Education. 2012; 12:1.

6. Pickering JD, Bickerdike SR. Medical student use of Facebook to support preparation for anatomy assessments. Anat Sci Educ. 2017; 10:205-14.

7. Hennessy CM, Kirkpatrick E, Smith CF, Border S. Social media and anatomy education: Using twitter to enhance the student learning experience in anatomy. Anat Sci Educ. 2016; 9:505-15.

8. Park KH, Park JH, Kim S, Rhee J, Kim JH, Ahn YJ, et al. Students' perception of the educational environment of medical schools in Korea: findings from a nationwide survey. Korean J Med Educ. 2015; 27:117-30.

9. Küçük S, Kapakin S, Göktaş Y. Learning anatomy via mobile augmented reality: Effects on achievement and cognitive load. Anat Sci Educ. 2016; 9:411-21.

10. Evans DJ, Cuffe T. Near-peer teaching in anatomy: An approach for deeper learning. Anat Sci Educ. 2009; 2:227-33.

11. Singh S. Near-peer role modeling: The fledgling scholars education paradigm. Anat Sci Educ. 2010; 3:50-1.

12. Durán CEP, Bahena EN, Rodríguez MdlÁG, Baca GJ, Uresti AS, Elizondo-Omaña RE, et al. Near-peer teaching in an anatomy course with a low faculty-to-student ratio. Anat Sci Educ. 2012; 5:171-6. 